\title{
Un ciclo de mejora docente para la formación de maestros y maestras en Didáctica de las Ciencias Sociales
}

ELISA NAVARRO-MEDINA

Universidad de Sevilla

Didáctica de las Ciencias

Experimentales y Sociales

enavarro5@us.es

ORCID: https://orcid.org/0000-0001-5523-7097

D.O.I.: http://dx.doi.org/10.12795/JDU.2018.i01.51

Pp.: 898-921

\section{Resumen:}

El Ciclo de Mejora Docente (CMD) presentado en esta comunicación aborda el desarrollo competo de los contenidos que se trabajan en la asignatura de Didáctica de las Ciencias Sociales del Grado de Educación Primaria. A través de cinco problemas de trabajo interrelacionados y encadenados, se pone el foco de atención en la necesidad de explicitar los contenidos que se pretenden enseñar, de planificar secuencias de actividades para trabajarlos y de tener en cuenta las ideas de los estudiantes para conocer la evolución de sus aprendizajes y sus obstáculos durante el proceso. 
Palabras clave: Didáctica de las Ciencias Sociales, Grado en Educación Primaria, docencia universitaria, experimentación docente universitaria.

\section{Breve descripción del contexto}

La asignatura en la que he implementado este año mi CMD es en Didáctica de las Ciencias Sociales, del tercer curso del Grado de Educación Primaria. Cada clase se organiza en dos sesiones de hora y veinte con el grupo completo y una sesión de hora y veinte con cada mitad del grupo. La asignatura consta de cinco bloques o problemas de trabajo (Bain, 2007) que abordan las cuestiones básicas del perfil profesional del maestro o maestra de educación primaria: finalidades de la enseñanza de las Ciencias Sociales, las ideas de los estudiantes, los contenidos y la metodología de enseñanza y los procesos de evaluación. Estas cuestiones básicas sirven a su vez para que los estudiantes vayan aplicando los conocimientos adquiridos a la elaboración de una propuesta didáctica de enseñanza, de forma que tanto el contenido teórico como práctico de la asignatura debe revertir en el diseño de un material curricular.

La asistencia de los 70 estudiantes que conforman el grupo ha sido regular durante toda la aplicación del CMD. Es de destacar la alta participación del grupo, que se ha traducido, no sólo en responder a las actividades planteadas, sino también en proponer, sugerir y pedir otras complementarias que les ayudasen a profundizar en su formación.

Jornadas de Formación e Innovación Docente del Profesorado | № 1 (2018) Esta obra se distribuye con la licencia Creative Commons 


\section{Diseño previo del Ciclo de Mejora Docente}

\section{Modelo metodológico consolidado}

Desde que hice el Curso General de Docencia Universitaria en la Universidad de Sevilla en el año 2013, Llevo aplicando cambios y mejoras en esta asignatura, consiguiendo definir un modelo metodológico estable (García y Porlán, 2017) -aunando mi modelo posible e ideal- (Navarro-Medina, 2017):

1. Plantear un gran problema de investigación. 2. Testear las ideas previas de los alumnos para conocer su punto de partida (con las mismas cuestiones al final). 3. Vincular sus ideas a un gran problema o cuestión que han de resolver a partir de las respuestas a los distintos subproblemas o subpreguntas. 4. Elaborar una secuencia de actividades investigativa (planteamiento de hipótesis, búsqueda y contraste de información, conclusiones) para cada subproblema, donde los estudiantes trabajen niveles de formulación del contenido de lo simple a lo complejo, con abundantes recursos diversos. 5. Entre subproblema y subproblema, testear las concepciones de los estudiantes, para conocer cómo va evolucionando su proceso de aprendizaje y poder detectar las dificultades que se van encontrando. 6 . Finalizar dando respuesta al gran problema de investigación y conociendo los aprendizajes alcanzados por los estudiantes. 7. Encadenar los ciclos de mejora, de forma que para cada problema trabajado se puedan ir detectando los obstáculos de aprendizaje de los estudiantes y se puedan ir retomando en los ciclos de mejora siguientes (p. 607).

Sin embargo, y tras el análisis de la experiencia del año pasado es necesario determinar algunas cuestiones que incluyo en este nuevo ciclo: 1. Reformular los problemas y 
las secuencias de actividades asociadas para que verdaderamente cumplan con los objetivos que me planteo, por lo que me he visto obligada a modificar los mapas de contenidos eliminando contenido que era secundario. 2. Incluir, dentro de las secuencias de actividades, propuestas para superar los obstáculos que se han manifestado a partir de la evaluación de los aprendizajes y del diseño realizado, de forma que pueda ir teniendo identificados aquellos elementos sobre los que poner la atención en años sucesivos y tener planificadas actividades que ayuden a superarlos. Para ello, he modificado algunas de las actividades de contraste de años anteriores.

\section{Mapas de contenidos y problemas}

Como he comentado anteriormente, la asignatura está dividida en cinco problemas-preguntas principales, que para mi son las cuestiones estructuradoras de la disciplina (véase figura 1). Estos cinco problemas llevan asociados algunos problemas o cuestiones que se trabajan durante la asignatura y que considero fundamentales, en un intento de ver esos contenidos desde la perspectiva macro, de aquello que verdaderamente sea lo más relevante que mis estudiantes deben de aprender. Los problemas generales que se han trabajado, en tono de azul más oscuro, los he desarrollado de forma más amplia en cada uno de los ciclos de mejora encadenados que he trabajado.

Jornadas de Formación e Innovación Docente del Profesorado | № 1 (2018) Esta obra se distribuye con la licencia Creative Commons 


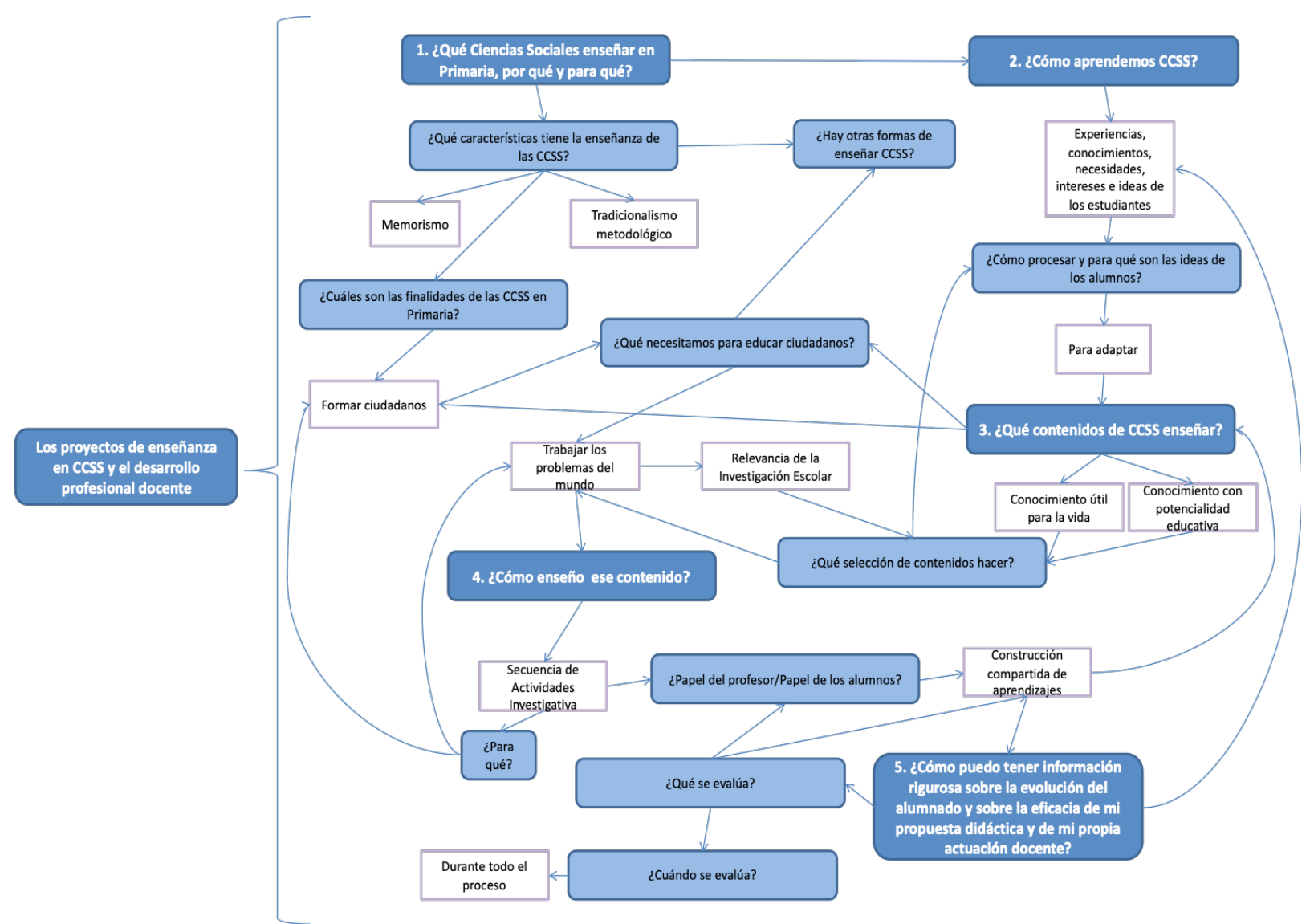

Figura 1. Mapa de contenidos general de la asignatura.png

Jornadas de Formación e Innovación Docente del Profesorado | № 1 (2018)

(c) E) Esta obra se distribuye con la licencia Creative Commons

(c) $(7) \Theta$ Reconocimiento-NoComercial-SinObraDerivada

Internacional (CC BY-NC-ND 4.0.) 
A continuación, se especifica cada uno de los problemas de trabajo. Para no complejizar en exceso el mapa, he dado valores numéricos a los procedimientos y valores alfabéticos a las actitudes.

La leyenda de los procedimientos es la siguiente: 1. Análisis de casos. 2. Uso de NNTT para la enseñanza. 3. Técnicas de debate y exposición de ideas. 4. Análisis y discusión de texto. 5. Comentario crítico de vídeos. 6. Comentario crítico de materiales didácticos. 7. Diseño de materiales didácticos. 8. Experimentación práctica. 9. Capacidad de cambio/ evolución de ideas.

La leyenda de las actitudes es la siguiente: a. Toma de conciencia. b. Autorreflexión. c. Actitud crítica hacia la realidad. d. Valoración de otras propuestas didácticas. e. Resolución activa ante problemáticas educativas. f. Predisposición al cambio/evolución de las ideas propias.

Jornadas de Formación e Innovación Docente del Profesorado | № 1 (2018) Esta obra se distribuye con la licencia Creative Commons 


\section{4}

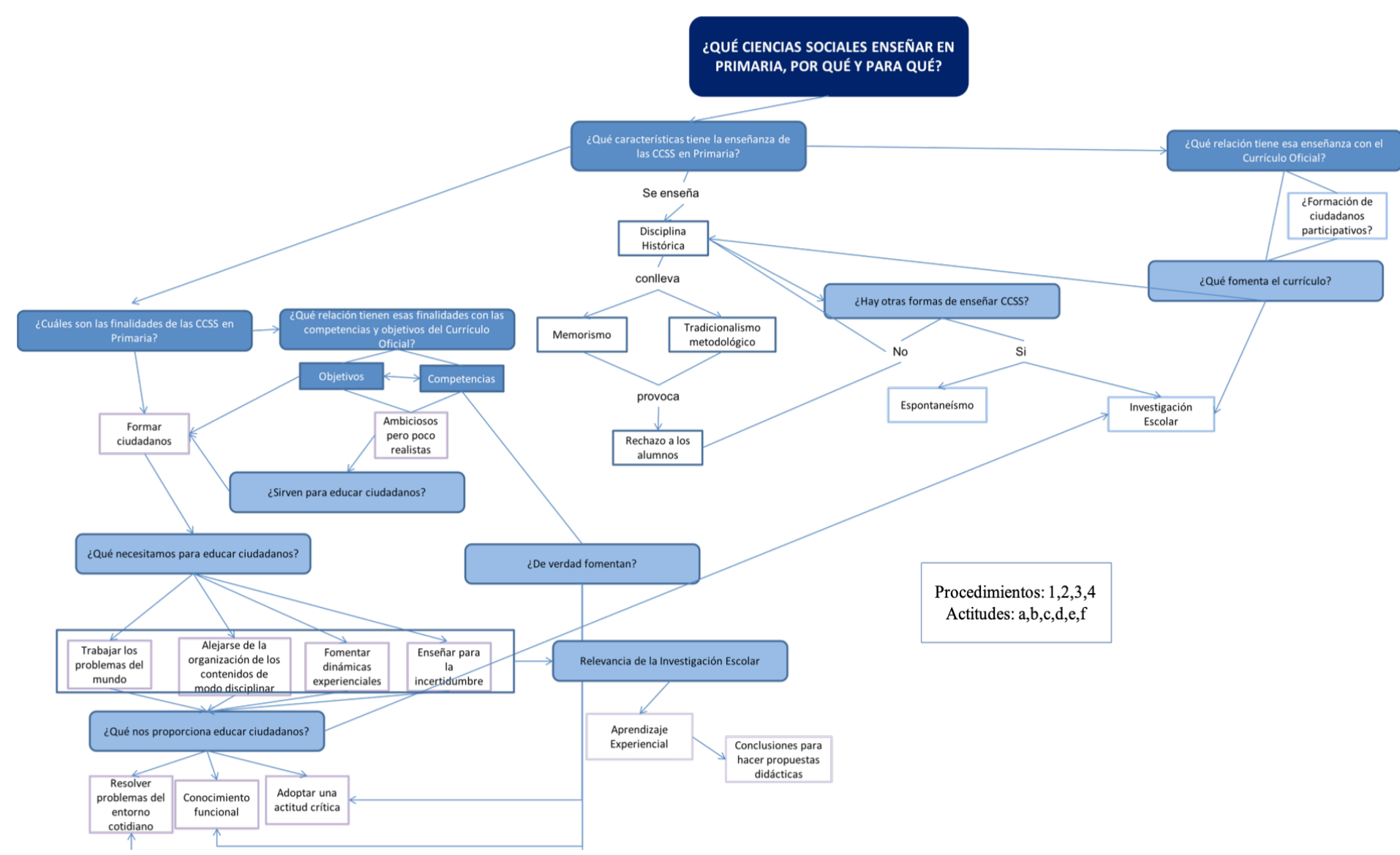

Figura 2. Mapa de contenido del problema 1.png

Jornadas de Formación e Innovación Docente del Profesorado | № 1 (2018)

(c) E) Esta obra se distribuye con la licencia Creative Commons

(c) $(7) \Theta$ Reconocimiento-NoComercial-SinObraDerivada

Internacional (CC BY-NC-ND 4.0.) 


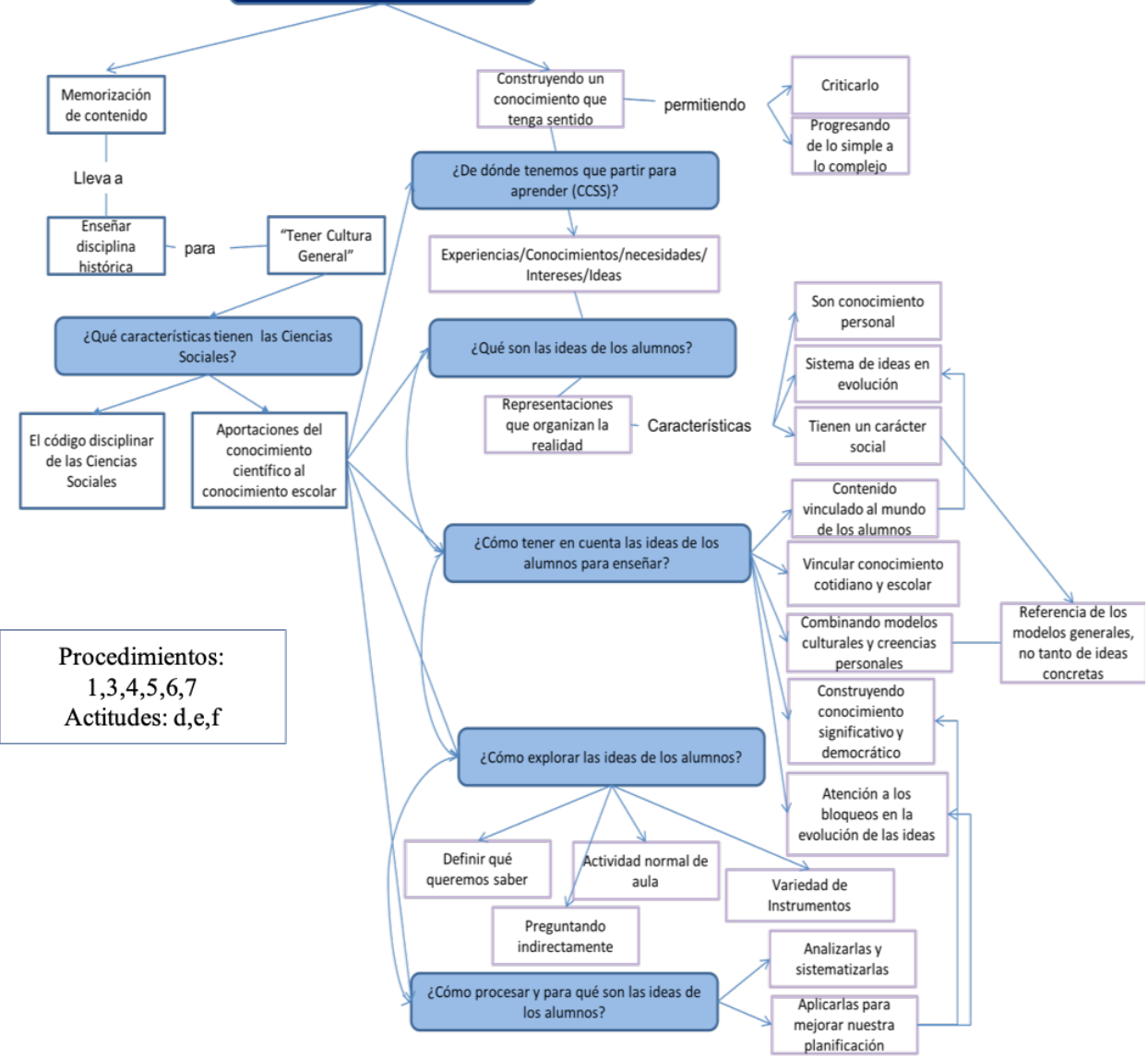

Figura 3. Mapa de contenidos del problema 2 


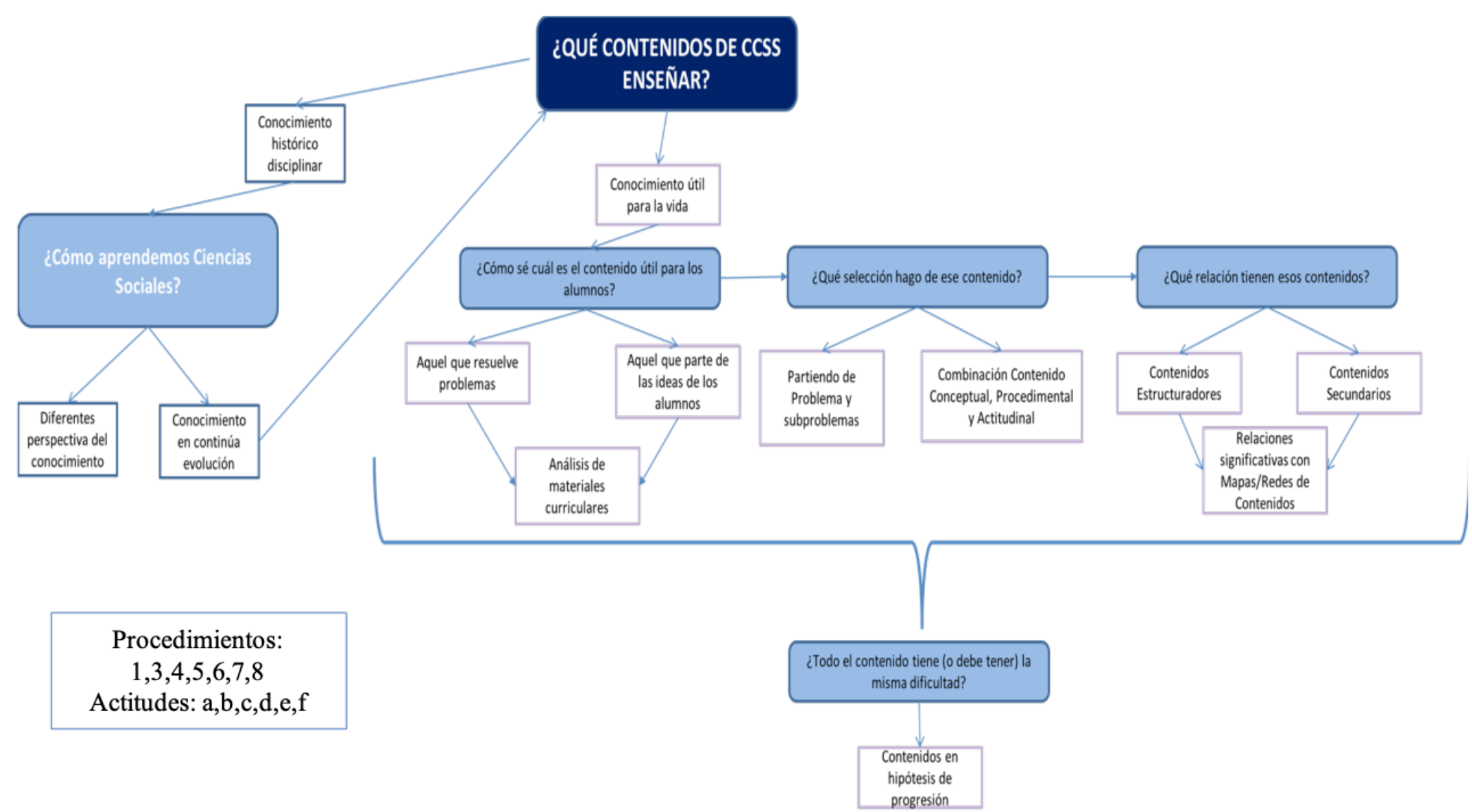

Figura 4. Mapa de contenidos del problema 3

Jornadas de Formación e Innovación Docente del Profesorado | № 1 (2018) (c) (i) $\odot$ Esta obra se distribuye con la licencia Creative Commons Internacional (CC BY-NC-ND 4.0.) 
¿CÓMO ENSEÑO ESE CONTENIDO?

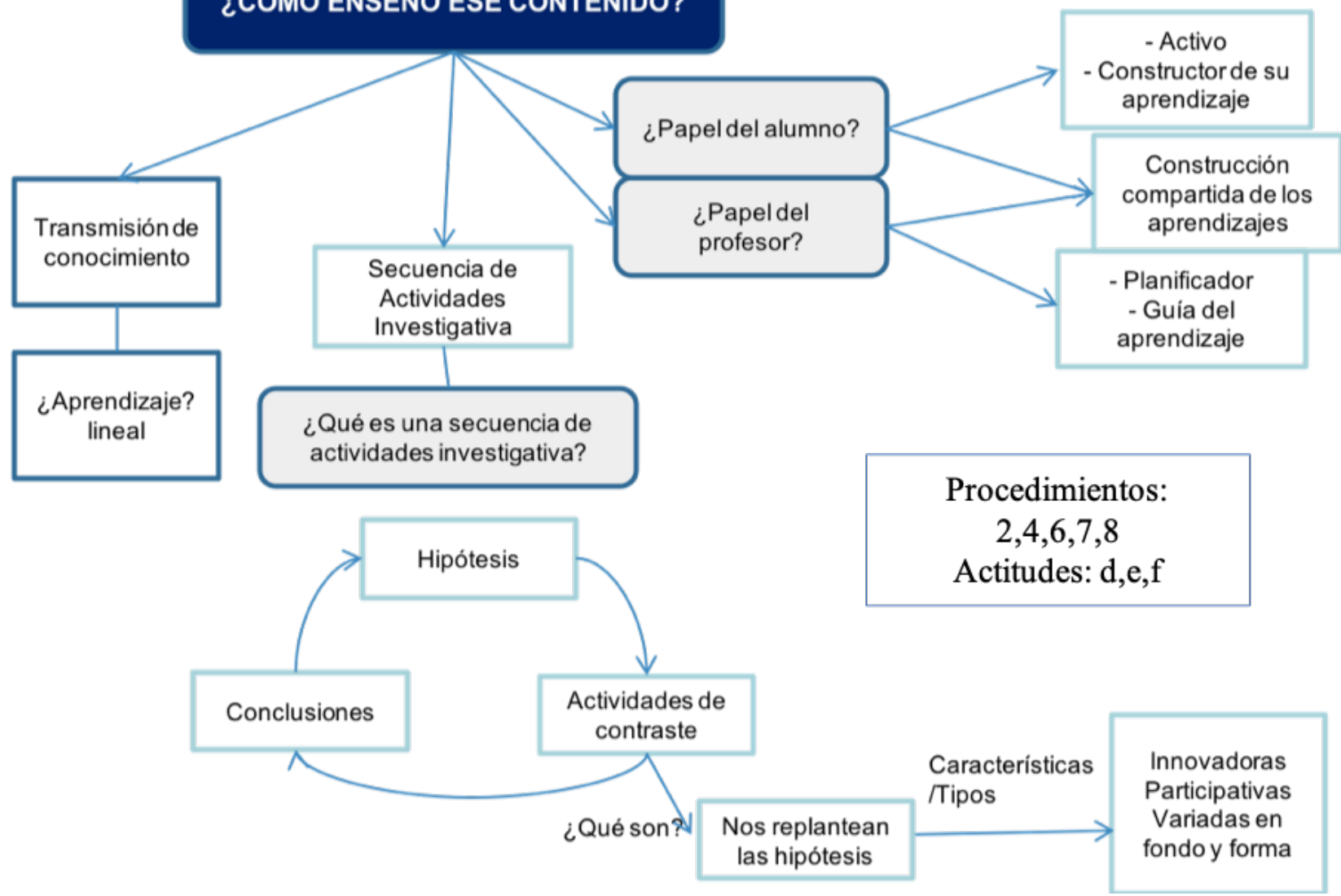

Figura 5. Mapa de contenidos del problema 4

Jornadas de Formación e Innovación Docente del Profesorado | № 1 (2018)

(c) $($ ) $\Theta$ Esta obra se distribuye con la licencia Creative Commons 


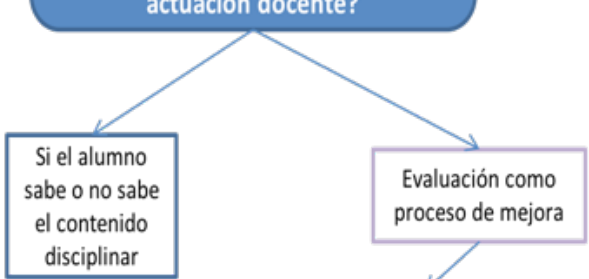

¿Qué se evalúa?

Procedimientos: $6,7,8$

Actitudes: d,e,f

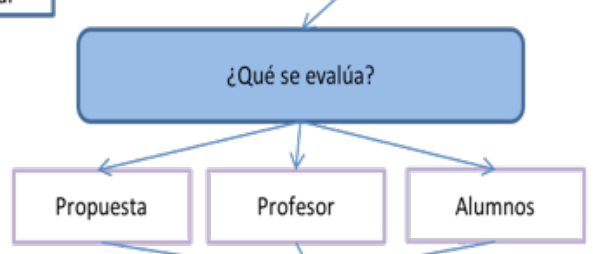

¿Cuándo se evalúan?

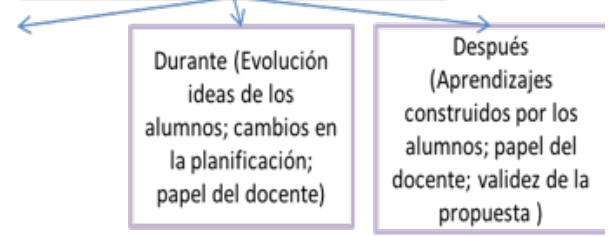

¿Cómo se evalúa?

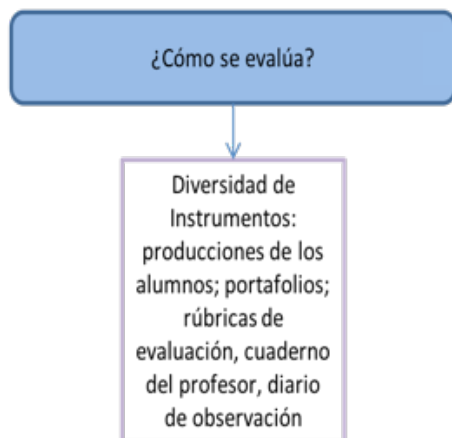

Figura 6. Mapa de contenidos del problema 5

\section{Cuestionario inicial y final para los estudiantes y secuencia de actividades}

Para recoger la evolución individual de las ideas de los estudiantes, se han ido pasando distintos cuestionarios, 
al inicio y al final de cada problema, recogiendo aquellas cuestiones que considero más relevantes en cada uno de ellos. Las preguntas han sido las siguientes:

Problema 1. ¿Qué Ciencias Sociales enseñar en Primaria, por qué y para qué?: 1. ¿Qué características tiene la enseñanza de las Ciencias Sociales en Primaria? (por ejemplo, papel de docente y estudiantes, contenidos, actividades, evaluación, etc.)? ¿Podría tener otras? ¿Cuáles serían? 2. ¿Cuáles son las finalidades de las Ciencias Sociales en Primaria? ¿Para qué deben servir? 3. ¿Qué cosas hay que enseñar a los alumnos para educarlos como ciudadanos? 4. ¿Es posible que un maestro/a sea también un investigador de su aula? Justifica tu respuesta. 5. ¿Qué conoces de la propuesta de currículo que hace la LOMCE para el área de Ciencias Sociales en Educación Primaria?

Problema 2. ¿Cómo aprendemos Ciencias Sociales?: 1. ¿Cómo aprendemos Ciencias Sociales? 2. ¿Qué características tienen las Ciencias Sociales como disciplina escolar? 3. ¿De dónde tenemos que partir para aprender? 4. ¿Qué son las ideas de los alumnos? 5. ¿Cómo tener en cuenta las ideas de los alumnos para enseñar? 6. ¿Cómo explorar las ideas de los alumnos? 7. ¿Cómo procesar y para qué son las ideas de los alumnos?

Problema 3. ¿Qué contenidos de Ciencias Sociales enseñar?: 1. ¿Qué contenidos de CCSS enseñar? 2. ¿Cómo sé cuál es el contenido útil para los alumnos? 3. ¿Qué selección hago de ese contenido? 4. ¿Qué relación tienen esos contenidos?

Problema 4. ¿Cómo enseño ese contenido?: 1. ¿Cómo enseño el contenido que quiero que mis alumnos aprendan? 2. ¿Qué papel corresponde a los alumnos? 3. ¿Qué papel corresponde al profesor? 4. ¿Qué es una secuencia de actividades investigativa?

Problema 5. ¿Cómo puedo obtener información rigurosa sobre la evolución del alumnado, y sobre la eficacia de mi propuesta didáctica y de mi propia actuación docente?: 1. ¿Cómo podemos obtener información rigurosa sobre la 
evolución del alumnado y sobre la eficacia de nuestra propuesta didáctica y de nuestra propia actuación docente? 2. ¿Qué se evalúa? 3. ¿Cuándo se evalúa? 4. ¿Cómo se evalúa?

\section{Tabla 1}

Secuencia de actividades del problema 2. ¿Cómo aprendemos Ciencias Sociales?

\begin{tabular}{|l|l|l|}
\hline \multicolumn{3}{|l|}{ SESIÓN 1. Presentación de problema } \\
\hline Tipo de Actividad & Descripción & Tiempo \\
\hline $\begin{array}{l}\text { Actividad de Inicio } \\
\text { de Sesión (IAS) }\end{array}$ & $\begin{array}{l}\text { Se retoman las ideas de la sesión anterior: } \\
\text { las finalidades de las CCSS y la necesidad } \\
\text { de enseñar un contenido que permitiese } \\
\text { formar ciudadanos críticos y participativos. }\end{array}$ & $\begin{array}{l}20 \\
\text { minutos }\end{array}$ \\
\hline $\begin{array}{l}\text { Cuestionario de } \\
\text { ideas previas (CIP) }\end{array}$ & $\begin{array}{l}\text { Cuestionario de ideas previas del problema } \\
\text { 2, a través de la plataforma Socrative. }\end{array}$ & $\begin{array}{l}40 \\
\text { minutos }\end{array}$ \\
\hline $\begin{array}{l}\text { Actividad de } \\
\text { puesta en común } \\
\text { de ideas (APCI) }\end{array}$ & $\begin{array}{l}\text { Se ponen en común las ideas iniciales so- } \\
\text { bre el problema a trabajar y se extraen con- } \\
\text { clusiones de grupo. }\end{array}$ & $\begin{array}{l}20 \\
\text { minutos }\end{array}$ \\
\hline
\end{tabular}

SESIÓN 2. PROBLEMA: ¿Cómo aprendemos Ciencias Sociales? Subproblema: ¿Qué características tienen las Ciencias Sociales como disciplina escolar?

\begin{tabular}{|c|c|c|}
\hline $\begin{array}{l}\text { Tipo de } \\
\text { Actividad }\end{array}$ & Descripción & Tiempo \\
\hline $\begin{array}{l}\text { Actividad de } \\
\text { Planteamiento } \\
\text { de problema/ } \\
\text { Actividad de } \\
\text { Contraste } 1 \\
\text { (APP/AC1) }\end{array}$ & $\begin{array}{l}\text { Lectura del texto: ¿Cómo se gestó la Histo- } \\
\text { ria escolar? Un ejemplo. Cuesta Fernández, } \\
\text { R. (1998). Rasgos arquetípicos de los manua- } \\
\text { les y programas de Historia. Doc. extractado } \\
\text { de: R. Cuesta Fernández, Clío en las aulas. La } \\
\text { enseñanza de la Historia en España entre re- } \\
\text { formas, ilusiones y rutinas. Madrid: Akal (pp. } \\
\text { 28-32). Extraer tres ideas principales. }\end{array}$ & $\begin{array}{l}1 \text { ho- } \\
\text { ras } 20 \\
\text { minutos }\end{array}$ \\
\hline
\end{tabular}

Jornadas de Formación e Innovación Docente del Profesorado | № 1 (2018) Esta obra se distribuye con la licencia Creative Commons Reconocimiento-NoComercial-SinObraDerivada $\quad 4.0$ Internacional (CC BY-NC-ND 4.0.) 
SESIÓN 3. PROBLEMA: ¿Cómo aprendemos Ciencias Sociales? Subproblema: ¿Qué características tienen las Ciencias Sociales como disciplina escolar?

\begin{tabular}{|l|l|l|}
\hline Tipo de Actividad & Descripción & Tiempo \\
\hline AC2 & $\begin{array}{l}\text { Lectura del texto: Aportaciones del conoci- } \\
\text { miento científico de las Ciencias Sociales al } \\
\text { conocimiento escolar. Domínguez Garrido, } \\
\text { C. (Coord.) (2004). Didáctica de las Ciencias } \\
\text { Sociales para Primaria. Madrid: Pearson } \\
\text { Educación. Selección de Cap. 2: Concepto y } \\
\text { campo epistemológico de las Ciencias So- } \\
\text { ciales (II) (págs. 31-39). Extraer tres ideas } \\
\text { principales. }\end{array}$ & \\
\hline AC3 & $\begin{array}{l}\text { Definición, en pequeño grupo, de las prin- } \\
\text { cipales caracteristicas que tienen las Cien- } \\
\text { cias Sociales como disciplina escolar. }\end{array}$ & $\begin{array}{l}20 \\
\text { minutos }\end{array}$ \\
\hline
\end{tabular}

SESIÓN 4. PROBLEMA: ¿Cómo aprendemos Ciencias Sociales? Subproblema: ¿Qué características tienen las Ciencias Sociales como disciplina escolar?

\begin{tabular}{|l|l|l|}
\hline Tipo de Actividad & Descripción & Tiempo \\
\hline AC4 & $\begin{array}{l}\text { Aportaciones de las diversas Ciencias So- } \\
\text { ciales a la temática o problema de la uni- } \\
\text { dad didáctica o proyecto elegido para } \\
\text { diseñar. Realización de un mapa de con- } \\
\text { tenidos individual con el contenido a } \\
\text { enseñar. }\end{array}$ & $\begin{array}{l}\text { y } 20 \\
\text { minutos }\end{array}$ \\
\hline
\end{tabular}

SESIÓN 5 y 6. PROBLEMA: ¿Cómo aprendemos Ciencias Sociales? Subproblema: ¿Qué características tienen las Ciencias Sociales como disciplina escolar?

\begin{tabular}{|l|l|l|}
\hline Tipo de Actividad & Descripción & Tiempo \\
\hline $\begin{array}{l}\text { Actividad Conclu- } \\
\text { sión Subproblema } \\
\text { (ACSub) }\end{array}$ & $\begin{array}{l}\text { Negociación del mapa de contenidos en } \\
\text { grupo: elaboración del mapa de conteni- }\end{array}$ & $\begin{array}{l}\text { y } 20 \\
\text { dos final del grupo. }\end{array}$ \\
\hline
\end{tabular}

Jornadas de Formación e Innovación Docente del Profesorado | № 1 (2018) cc) (†) $\odot$ Esta obra se distribuye con la licencia Creative Commons Reconocimiento-NoComercial-SinObraDerivada $\quad 4.0$ Internacional (CC BY-NC-ND 4.0.) 
SESIÓN 7. PROBLEMA: ¿Cómo aprendemos Ciencias Sociales? Subproblemas: ¿De dónde tenemos que partir para aprender?, ¿Qué son las ideas de los alumnos?, ¿Cómo tener en cuenta las ideas de los alumnos para enseñar?, ¿Cómo explorar las ideas de los alumnos?

\begin{tabular}{|l|l|l|}
\hline Tipo de Actividad & Descripción & Tiempo \\
\hline AC1 & $\begin{array}{l}\text { Vídeo: “Sheldom enseña física a } \\
\text { Penny", analizando los aspectos } \\
\text { más relevantes del tipo de aprendi- } \\
\text { zaje. Debate sobre cómo debe ser el } \\
\text { aprendizaje de los seres humanos. }\end{array}$ & $\begin{array}{l}15 \\
\text { minutos }\end{array}$ \\
\hline AC2 & $\begin{array}{l}\text { Vídeo: “Nacidos para aprender". De- } \\
\text { bate de las características que debe } \\
\text { tener el aprendizaje para que sea un } \\
\text { aprendizaje funcional }\end{array}$ & $\begin{array}{l}20 \\
\text { minutos }\end{array}$ \\
\hline AC3 & $\begin{array}{l}\text { Debate sobre formas de explorar las } \\
\text { ideas de los alumnos. Contraste con } \\
\text { ejemplos de cuestionarios (tipos de } \\
\text { preguntas y respuestas). }\end{array}$ & $\begin{array}{l}30 \\
\text { minutos }\end{array}$ \\
\hline AC4 & $\begin{array}{l}\text { Lectura del texto: García Pérez, F.F. } \\
\text { (2003). ¿Por qué es necesario que los } \\
\text { profesores tengamos en cuenta las } \\
\text { ideas de los alumnos? Extraer tres } \\
\text { ideas principales. }\end{array}$ & $\begin{array}{l}10 \\
\text { minutos }\end{array}$ \\
\hline
\end{tabular}

SESIÓN 8-9. PROBLEMA: ¿Cómo aprendemos Ciencias Sociales? Subproblemas: ¿De dónde tenemos que partir para aprender?, ¿Qué son las ideas de los alumnos?, ¿Cómo tener en cuenta las ideas de los alumnos para enseñar?, ¿Cómo explorar las ideas de los alumnos?

\begin{tabular}{|l|l|l|}
\hline Tipo de Actividad & Descripción & Tiempo \\
\hline AC5 & $\begin{array}{l}\text { Trabajo preparatorio de una propuesta de } \\
\text { exploración de ideas de los estudiantes en } \\
\text { base a la problemática seleccionada y al } \\
\text { mapa de contenidos realizado por el grupo. } \\
\text { Selección del grupo de estudiantes que par- } \\
\text { ticiparán en la exploración y recogida de los } \\
\text { datos. }\end{array}$ & \\
minutos \\
\end{tabular}

Jornadas de Formación e Innovación Docente del Profesorado | № 1 (2018) Esta obra se distribuye con la licencia Creative Commons Reconocimiento-NoComercial-SinObraDerivada Internacional (CC BY-NC-ND 4.0.) 
SESIÓN 10. PROBLEMA: ¿Cómo aprendemos Ciencias Sociales? Subproblemas: ¿De dónde tenemos que partir para aprender?, ¿Qué son las ideas de los alumnos?, ¿Cómo tener en cuenta las ideas de los alumnos para enseñar?, ¿Cómo explorar las ideas de los alumnos?

\begin{tabular}{|l|l|l|}
\hline $\begin{array}{l}\text { Tipo de } \\
\text { Actividad }\end{array}$ & Descripción & Tiempo \\
\hline AC6 & $\begin{array}{l}\text { Análisis básicos de los resultados obtenidos } \\
\text { a partir de la aplicación del instrumento de } \\
\text { ideas a los estudiantes. }\end{array}$ & $\begin{array}{l}1 \text { hora y 20 } \\
\text { minutos }\end{array}$ \\
\hline
\end{tabular}

SESIÓN 11. PROBLEMA: ¿Cómo aprendemos Ciencias Sociales? Subproblemas: ¿De dónde tenemos que partir para aprender?, ¿Qué son las ideas de los alumnos?, ¿Cómo tener en cuenta las ideas de los alumnos para enseñar?, ¿Cómo explorar las ideas de los alumnos?

\begin{tabular}{|l|l|l|}
\hline $\begin{array}{l}\text { Tipo de } \\
\text { Actividad }\end{array}$ & Descripción & Tiempo \\
\hline ACSub & $\begin{array}{l}\text { Elaboración de un primer borrador de mapa } \\
\text { de contenidos y propuesta justificada de ex- } \\
\text { ploración de ideas los estudiantes, aplicando } \\
\text { dicho instrumento y analizando sintética- } \\
\text { mente los resultados obtenidos. }\end{array}$ & $\begin{array}{l}1 \text { hora } \\
\text { minutos } \\
\text { mina }\end{array}$ \\
\hline $\begin{array}{l}\text { Actividad } \\
\text { de Conclu- } \\
\text { sión (no } \\
\text { presencial) }\end{array}$ & $\begin{array}{l}\text { Reflexión sobre lo aprendido contestando a } \\
\text { las preguntas del problema 2. }\end{array}$ & \\
\hline
\end{tabular}

\section{Aplicación del Ciclo de Mejora Docente}

\section{Relato resumido de las sesiones}

Las sesiones de trabajo en los dos problemas que se han implementado ha sido muy satisfactoria, aunque comento algunas cuestiones surgidas:

- Es necesario entrenar las formas de trabajo en el aula, pues en ocasiones, el trabajo en pequeño grupo no ha sido aprovechado para el desarrollo de la actividad de clase. Para solventar este problema, se ha negociado

Jornadas de Formación e Innovación Docente del Profesorado | № 1 (2018) Esta obra se distribuye con la licencia Creative Commons Reconocimiento-NoComercial-SinObraDerivada $\quad 4.0$ Internacional (CC BY-NC-ND 4.0.) 
con los estudiantes un Contrato de Trabajo, que consta de 5 puntos: 1. Las tareas propuestas en clase siempre empiezan marcando el objetivo a conseguir/su utilidad en la formación. 2. Las tareas tienen un tiempo establecido. La profesora hace una propuesta y los estudiantes la valoran a tenor de la carga de trabajo, aceptando o proponiendo cambios. 3. El trabajo en el aula se debe de aprovechar para plantear las dudas de la actividad. 4. La profesora pasa por los grupos para proponer mejoras que ayuden a avanzar en el trabajo. 5. El trabajo aprovechado en clase, que se mide a través de la observación que hace la profesora en el desarrollo de las clases y de la entrega de los materiales por parte de los estudiantes, se compensa en la nota final con hasta 3 puntos.

- La inclusión de las nuevas actividades de contraste, fruto de la evaluación realizada en el CMD del año anterior, ha resultado todo un acierto. Por ejemplo, para el problema 1, subproblemas ¿Qué relación tiene esa enseñanza con el Currículo Oficial de la LOMCE? ¿Qué relación tienen las finalidades de las CCSS con las competencias y objetivos de la LOMCE?, que en años anteriores no había alcanzado un grado de aprendizaje alto, pues los estudiantes no reflexionaban sobre el currículo, simplemente se limitaban a copiar objetivos y contenidos, ha permitido este año que los estudiantes debatan sobre el tipo de currículo oficial que se les presentan e incluso, hagan propuestas de cambios. Ello se ha conseguido porque a parte de pedirles que relacionen el currículo con la temática que estaban trabajando en el proyecto, se les ha pedido que hiciesen un listado de cuestiones a mejorar después del análisis realizado.

- He sido consciente de la necesidad de incluir actividades de conclusiones intermedias. Por ejemplo, en el problema 2 se suceden 6 actividades de contrate que, aún trabajando los mismos contenidos, se han vivido 
por los estudiantes como una sucesión de tareas independientes. Para el año que viene, creo que se puede prescindir de alguna actividad y en su lugar incluir actividades de conclusión que permitan a los estudiantes entender el sentido de la secuencia.

\section{Evaluación del aprendizaje de los estudiantes}

La evaluación de los aprendizajes de los estudiantes se ha realizado teniendo en cuenta 4 instrumentos: 1 . El análisis de las ideas previas y finales para cada uno de los problemas donde se pueden detectar los obstáculos de aprendizaje. 2. Los informes (individuales y grupales) que han entregado en cada uno de los problemas de trabajo y que recogen sus conclusiones y aplicaciones didácticas sobre lo que se hace en clase. 3. La asistencia y la participación en clase, acordada en el Contrato de Trabajo. 4. Un informe de conclusiones finales de la asignatura, donde a partir de tres cuestiones prácticas profesionales deben poner en funcionamiento los aprendizajes alcanzados.

En esta comunicación solo presento los datos del análisis de ideas a partir de los cuestionarios, pues tanto los informes como la asistencia y participación tienen un carácter de borrador y la evaluación que se hace de ellos se basa en anotaciones de mejora para que, con el trabajo de los siguientes problemas, vayan reformulando diversos aspectos y entreguen su versión definitiva al final de la asignatura.

Del análisis de los modelos mentales de los estudiantes en el problema 2. ¿Cómo aprendemos CCSS?, cuya evolución pre y post a la implementación del CMD podemos ver en la figura 7, podemos extraer algunas conclusiones generales:

- En general, el CMD ha ayudado a que las ideas de la mayoría de los estudiantes evolucionen de conocimientos más simples a conocimientos más complejos. Sin embargo, en casi todos los subproblemas trabajados, aún 
encontramos estudiantes que no alcanzan el nivel de desarrollo deseable, lo que indica la necesidad de establecer secuencias de actividades más adaptadas a todos los ritmos de aprendizaje.

- En varios de los subproblemas trabajados, los estudiantes muestran respuestas categorizadas en nivel 2 al inicio de la implementación del CMD. Preguntando a los estudiantes señalan que, en asignaturas del segundo curso (específicamente en Didáctica de las Ciencias Experimentales), dedicaron una buena parte del curso al trabajo en torno a las ideas. Este dato demuestra la necesaria coordinación que debe existir entre las asignaturas y me plantea el deber de reenfocar este problema de trabajo, eliminado aquellos contenidos ya trabajados y haciendo hincapié en otros elementos que no se abordan.

- Para el subproblema ¿Qué características tienen las CCSS como disciplina escolar? es necesario replantear la secuencia de actividades programada. En el desarrollo de las sesiones, y sobre todo tras el análisis de la evolución de las ideas, he sido consciente de que este subproblema tiene elementos complejos que los estudiantes no acaban de relacionar con su trabajo como maestros y maestras. Por ejemplo, entienden que es necesario trabajar en torno a problemas socialmente relevantes pero no acaban de construir una propuesta de conocimiento escolar que les permita hacerlo. En la mayoría de los casos se remiten constantemente al currículo y a elementos específicos de las disciplinas de Ciencias Sociales, sin acabar de construir la idea de conocimiento escolar como la unión entre el conocimiento científico, los problemas sociales y las ideas de los estudiantes (García, Porlán y Navarro, 2017). Se detecta, por tanto, un obstáculo importante de aprendizaje.

- Algo similar ocurre con el subproblema ¿Cómo tener en cuenta las ideas de los alumnos para enseñar? En este 
caso, el obstáculo de aprendizaje se centra en que los estudiantes no acaban de ver la finalidad práctica que tiene trabajar con las ideas de los alumnos durante todo el proceso de enseñanza, y cómo pueden detectar obstáculos y solventarlos. Esta cuestión requiere modificar la propuesta de actividades, incluyendo alguna actividad de contraste que ofrezca a los estudiantes la posibilidad de analizar datos reales de alumnos de primaria que le lleven a determinar dónde están esos obstáculos y qué deben de hacer para que puedan superarlos. Sin embargo, se plantea también un problema externo a la asignatura y es la escasa presencia de prácticas curriculares que permitan a los estudiantes poner en juego los aprendizajes que desarrollamos en las clases.

Jornadas de Formación e Innovación Docente del Profesorado | № 1 (2018) Esta obra se distribuye con la licencia Creative Commons 


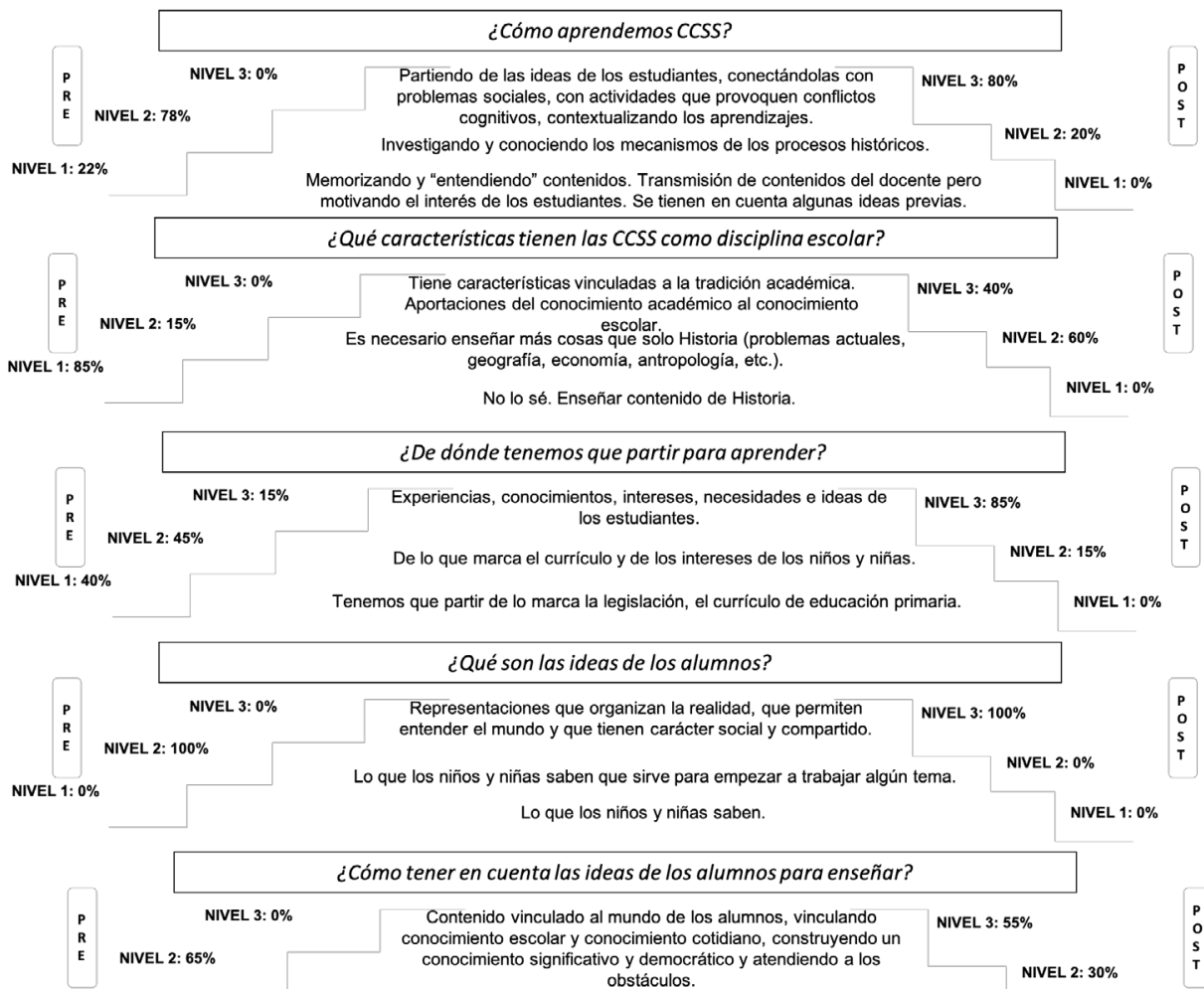

NIVEL $1: 35 \%$

obstáculos.
Nos dicen por dónde tenemos que empezar y los niños saben o no saben. Sirven para modificar nuestra unidad didáctica.

Preguntadles para conocer lo que saben de un tema.

¿Cómo explorar las ideas de los alumnos?

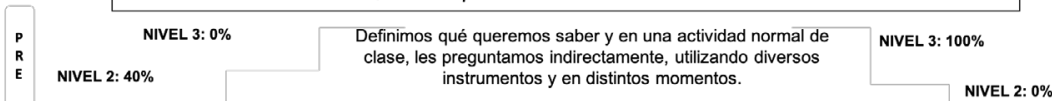

NIVEL 1: $60 \%$ Podemos utilizar distintos instrumentos y en varios momentos (al inicio, en medio o al final).

Con un cuestionario o una lluvia de ideas al principio de la unidad didáctica.

NIVEL 1: $0 \%$ Cómo procesary para qué son las ideas de los alumnos?

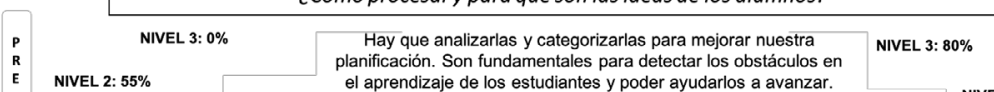

NIVEL 1: 45\% aprendizaje de los estudiantes y poder ayudarlos a avanzar.

\section{NIVEL 2: $20 \%$}

NIVEL 1: $0 \%$

Vemos las respuestas y vemos los errores para modificarlos.

Figura 7. Escaleras de aprendizajes de los estudiantes.png 


\section{Evaluación del Ciclo de Mejora Docente}

\section{Aspectos de la experiencia que se pretenden incorporar a toda la práctica docente habitual.}

- Con respecto al mapa de contenidos y problemas: mantendría la mayor parte de los mapas realizados. Sin embargo, con respecto al problema 2 ¿Cómo aprendemos CCSS?, considero fundamental establecer procesos de coordinación con otros compañeros y asignaturas que permita que el contenido trabajado no se repita y utilizar lo que los estudiantes ya saben para poder profundizar en otros contenidos.

- Con respecto a la metodología y la secuencia de actividades: en general las secuencias de actividades han funcionado bien pero es necesario readaptar dos cosas: más actividades de conclusión en los subproblemas, que ofrezcan a los estudiantes una visión unitaria del trabajo realizado, y no una parcelación de este; y atender a los obstáculos de aprendizaje detectados fruto del estudio de las ideas, incluyendo actividades específicas para solventarlos.

- Con respecto a la evaluación y el seguimiento de las ideas de los estudiantes: en este curso he conseguido ser mucho más sistemática con el trabajo de las ideas, sin embargo, el número de estudiantes en clase (70) y el propio desarrollo de las sesiones, hacen que trabajar con ellas suponga una carga de trabajo importante. Esto me plantea la cuestión: ¿hay otras formas de trabajar con las ideas de los estudiantes que sea rigurosa pero que no conlleve un esfuerzo de trabajo tan amplio? Investigaré para el próximo curso.

Jornadas de Formación e Innovación Docente del Profesorado | № 1 (2018) Esta obra se distribuye con la licencia Creative Commons 


\section{Principios didácticos argumentados que han guiado la experiencia y que deben permanecer en el futuro.}

- Tener definidos mapas de contenidos y problemas de trabajo ayuda a discriminar lo importante de lo secundario y ofrecen la capacidad de moverte por el contenido de forma más libre.

- Las secuencias de actividades detalladas dan seguridad en los procesos de enseñanza-aprendizaje, y tener explicitados los contenidos ayuda a detectar los obstáculos de aprendizaje que tienen los estudiantes. Cuando una actividad no cumple con la función para la que había sido planificada, porque los estudiantes no avanzan en el conocimiento, es fácil cambiarla.

- El trabajo con los modelos mentales de los estudiantes es fundamental, no sólo como instrumento de evaluación de aprendizajes, sino como herramienta para detectar obstáculos que permitan hacer modificaciones tanto en contenidos como en secuencias de actividades.

- Valoro de forma importante los acuerdos a los que se llega con los estudiantes. Hacedles partícipes del proceso, no como algo impuesto, sino como una experiencia democrática, ayuda a su implicación en la asignatura y en el trabajo diario.

Jornadas de Formación e Innovación Docente del Profesorado | № 1 (2018) Esta obra se distribuye con la licencia Creative Commons 


\section{Referencias bibliográficas}

Bain, K. (2007). Lo que hacen los mejores profesores universitarios. Trad. Oscar Barberá. Valencia: Universitat de Valencia.

García, E., Porlán, R. y Navarro, E. (2017). Los fines y los contenidos de ensenanza. En R. Porlán (Coord.), Enseñanza Universitaria. Cómo mejorarla (pp. 55-72). Madrid: Editorial Morata.

García, F.F. y Porlán, R. (2017). Los principios didácticos y el modelo didáctico personal. En R. Porlán (Coord.), Enseñanza Universitaria. Cómo mejorarla (pp. 93-104). Madrid: Editorial Morata.

Navarro-Medina, E. (2017). ¿Qué Ciencias Sociales enseñar en primaria, por qué y para qué? Un ciclo de mejora en el Grado de Educacion Primaria de la Universidad de Sevilla. En R. Porlán y E. Navarro-Medina (Coords.), IV Jornadas de Docencia Universitaria (págs. 599-615). Sevilla: Instituto de Ciencias de la Educacion de la Universidad de Sevilla.

Jornadas de Formación e Innovación Docente del Profesorado | № 1 (2018) Esta obra se distribuye con la licencia Creative Commons 